\title{
Open-access synthetic spike-in mRNA-seq data for cancer gene fusions
}

\author{
Waibhav D Tembe ${ }^{1 *}$, Stephanie JK Pond ${ }^{2}$, Christophe Legendre ${ }^{1}$, Han-Yu Chuang ${ }^{2}$, Winnie S Liang ${ }^{1}$, Nancy E Kim², \\ Valerie Montel ${ }^{2}$, Shukmei Wong ${ }^{1}$, Timothy K McDaniel${ }^{2}$, David W Craig ${ }^{1}$ and John D Carpten ${ }^{1}$
}

\begin{abstract}
Background: Oncogenic fusion genes underlie the mechanism of several common cancers. Next-generation sequencing based RNA-seq analyses have revealed an increasing number of recurrent fusions in a variety of cancers. However, absence of a publicly available gene-fusion focused RNA-seq data impedes comparative assessment and collaborative development of novel gene fusions detection algorithms. We have generated nine synthetic poly-adenylated RNA transcripts that correspond to previously reported oncogenic gene fusions. These synthetic RNAs were spiked at known molarity over a wide range into total RNA prior to construction of next-generation sequencing mRNA libraries to generate RNA-seq data.

Results: Leveraging a priori knowledge about replicates and molarity of each synthetic fusion transcript, we demonstrate utility of this dataset to compare multiple gene fusion algorithms' detection ability. In general, more fusions are detected at higher molarity, indicating that our constructs performed as expected. However, systematic detection differences are observed based on molarity or algorithm-specific characteristics. Fusion-sequence specific detection differences indicate that for applications where specific sequences are being investigated, additional constructs may be added to provide quantitative data that is specific for the sequence of interest.
\end{abstract}

Conclusions: To our knowledge, this is the first publicly available synthetic RNA-seq data that specifically leverages known cancer gene-fusions. The proposed method of designing multiple gene-fusion constructs over a wide range of molarity allows granular performance analyses of multiple fusion-detection algorithms. The community can leverage and augment this publicly available data to further collaborative development of analytical tools and performance assessment frameworks for gene fusions from next-generation sequencing data.

Keywords: RNA-seq, Gene fusions, Cancer genomics

\section{Background}

Oncogenic fusion genes underlie the mechanism of several common cancers and also constitute or encode important diagnostic and therapeutic targets. Fusions may drive oncogenic growth by joining a proliferationinducing gene to an active promoter, by disrupting the function of tumor suppressor genes, or by creating novel functional products that rewire the biochemical pathways that regulate cellular division [1]. Research has led to identification of drugs that are currently used to target fusions in different malignancies. Examples include imatinib, tretinoin, and crizotinib, which target the $B C R-A B L$,

\footnotetext{
* Correspondence: wtembe@tgen.org

${ }^{1}$ Translational Genomics Research Institute (TGen), 445 N 5th Street, SUITE 600, Phoenix, AZ 85004, USA

Full list of author information is available at the end of the article
}

PML-RAR, and EML4-ALK fusion products associated with chronic myelogenous leukemia [2,3], acute promyelocytic leukemia [4-6], and non-small cell lung carcinoma [7-9], respectively. These established associations and clinical applications underscore the need to comprehensively and accurately detect fusions in cancer samples.

Next-generation sequencing technologies, particularly RNA sequencing (RNA-seq), have revealed an increasing number of recurrent fusions in a variety of cancers, and it is likely that their detection will have growing diagnostic and prognostic utility. As such, validating the laboratory and analysis methods to establish analytical parameters including the limit of detection, linearity, sensitivity, and specificity of fusion detection in tumor RNA specimens is critical for adoption in clinical research settings. For example, does a fusion transcript present at higher molarity 
(higher transcript abundance) correlate with higher number of fusion-supporting sequencing reads? Are there differences in detection algorithms' efficacy with respect to specific fusion sequence and independent of abundance? Answering such questions and establishing robust metrics is difficult due to the lack of publicly available RNA-seq data specifically generated to capture gene fusions.

We have developed a set of nine synthetic polyadenylated RNA transcripts that correspond to reported cancer fusion gene sequences (Figure 1 and Additional file 1: Table S1). These synthetic gene fusion RNA constructs (SGFRs) can be spiked at known concentrations into total RNA prior to mRNA library construction and barcoded to keep them separate from endogenous fusions. To demonstrate utility of these SGFRs, we performed a series of experiments and data analyses as described next.

\section{Methods}

Generation of synthetic gene fusion RNA (SGFR) constructs Sequences of nine transcripts containing oncogenic fusions were obtained from GenBank. Degenerate bases in the sequences were assigned a specific base and the final sequences can be found in the separate excel sheet. A T7 promoter sequence and AscI restriction enzyme site were added to the $5^{\prime}$ end of the sequence and a T3 and NotI sequence added to the 3 ' end of the sequence to allow for linearization and transcription in both directions (Figure 2). The sequence was synthesized and inserted into a pUCIDT vector by IDT (San Diego, CA). Lyophilized plasmids were resuspended in $40 \mu \mathrm{L}$ TE. $50 \mu \mathrm{L}$ aliquots of Transformax ${ }^{\mathrm{Tm}} \mathrm{EC} 100^{\mathrm{Tm}}$ Chemically Competent E. coli (Epicenter, Madison WI) were thawed on ice and transfected with $1 \mu \mathrm{L}$ (9.7-83.1 ng) of resuspended plasmid per the manufacturer's suggested protocols. Transformed cells were plated on prewarmed $100 \mu \mathrm{g} / \mathrm{mL}$ ampicillin plates and incubated at $37^{\circ} \mathrm{C}$ overnight (18 hours). One colony from each plate was used to inoculate $5 \mathrm{~mL} \mathrm{LB}$ broth (Teknova) containing $1 \times$ carbenicillin. Inoculated tubes were incubated overnight on a shaker at $37^{\circ} \mathrm{C}$. Plasmids were isolated using the Qiagen Spin Miniprep Kit. The sequence of the purified plasmids were validated with Sanger sequencing. Purified plasmids were quantitated using the UV absorbance, then linearized with NotI-HF ${ }^{\text {тм }}$ (New England Biolabs) at $37^{\circ} \mathrm{C}$ for 4 hours. Linearized plasmids were gel purified on a $0.8 \%$ agarose gel. Linear DNA was excised from the gels and purified using QIAquick Gel Extraction Kit and ethanol precipitated. DNA was transcribed to RNA using MegaScript ${ }^{\oplus}$ T7 Kit (Invitrogen) followed by poly(A) tailing using the Poly(A) Tailing Kit (Life Technologies) according to the manufacturer recommended protocols. Poly-A tailed RNA was cleaned up using MEGAclear ${ }^{\mathrm{TM}} \mathrm{Kit}$ (Life Technologies, cat\#AM1908) and ethanol precipitated in aliquots for long-term storage.

\section{RNA sequencing}

RNA aliquots were washed in $70 \%$ ice cold ethanol, resuspended in $50 \mu \mathrm{L}$ TE buffer $(10 \mathrm{mM}$ Tris- $\mathrm{HCl} \mathrm{pH}$ 8.0, $1 \mathrm{mM}$ EDTA), then quantitated using UV absorption. 2.2 ng of each RNA spike were pooled in a PCR plate, and the volume was brought up to $50 \mu \mathrm{L}$ with RNase free water. A cDNA library was prepared using TruSeq Stranded mRNA LT Sample Prep Kit (Illumina ${ }^{\circledR}$ cat\# RS-122-2101) and sequenced on an Illumina MiSeq to confirm the sequences of the mRNA transcripts as a final QC step. Fresh aliquots of RNA were taken from storage, washed with $70 \%$ ice cold ethanol, resuspended in $1 \times \mathrm{TE}$, and quantitated using RiboGreen (Invitrogen). RNA spikes were mixed together to create a high concentration pool with $40 \mathrm{nM}$ of each spike. This pool was diluted and titrated into to $1 \mu \mathrm{g}$ aliquots of COLO-829 total RNA (ATCC 1974). cDNA libraries were prepared using the TruSeq Stranded mRNA LT Sample Prep Kit (Illumina ${ }^{\oplus}$ cat\# RS-122-2101) following the manufacturer's protocol. The resulting libraries were sequenced on the Illumina HiSeq2500 in Rapid Run mode using paired end reads with 101 cycles in each read.

In summary, equimolar amounts of all nine SGFRs were pooled together and this pool was titrated into total RNA from the melanoma cell line COLO-829 [10] at ten different abundances. Each SGFR abundance pool was prepared in duplicate. Libraries were prepared for sequencing using the Illumina TruSeq Stranded

\begin{tabular}{|c|c|c|c|c|c|}
\hline $\begin{array}{c}\text { Fusion } \\
\text { (5'-3'genes) }\end{array}$ & $\begin{array}{c}\text { Sequence } \\
\text { Source }\end{array}$ & $\begin{array}{c}\text { 5' gene } \\
\text { length }\end{array}$ & $\begin{array}{c}\text { 3' gene } \\
\text { length }\end{array}$ & $\begin{array}{c}\text { Total } \\
\text { length }\end{array}$ & $\begin{array}{c}\text { \%GC } \\
\text { content }\end{array}$ \\
\hline EWS-ATF1 & JN624779 (NCBI) & 793 & 305 & 1098 & $50.27 \%$ \\
\hline TMPRSS2-ETV1 & TGen/lllumina & 219 & 1011 & 1230 & $52.03 \%$ \\
\hline EWS-FLI1 & TGen/lllumina & 793 & 638 & 1431 & $55.56 \%$ \\
\hline NTRK3-ETV6 & TGen/lllumina & 1009 & 893 & 1902 & $55.47 \%$ \\
\hline CD74-ROS1 & EU236945 (NCBI) & 625 & 1487 & 2112 & $48.20 \%$ \\
\hline HOOK3-RET & DQ104207(NCBI) & 1122 & 1209 & 2331 & $48.13 \%$ \\
\hline EML4-ALK & AB663645.1 (NCBI) & 1731 & 1659 & 3390 & $49.03 \%$ \\
\hline AKAP9-BRAF & TGen/lllumina & 3318 & 1161 & 4479 & $37.22 \%$ \\
\hline BRD4-NUT & AY166680 (NCBI) & 534 & 3383 & 3917 & $54.74 \%$ \\
\hline
\end{tabular}

Figure 1 Summary of nine synthetic fusion gene transcripts, excluding the poly-A tail. 


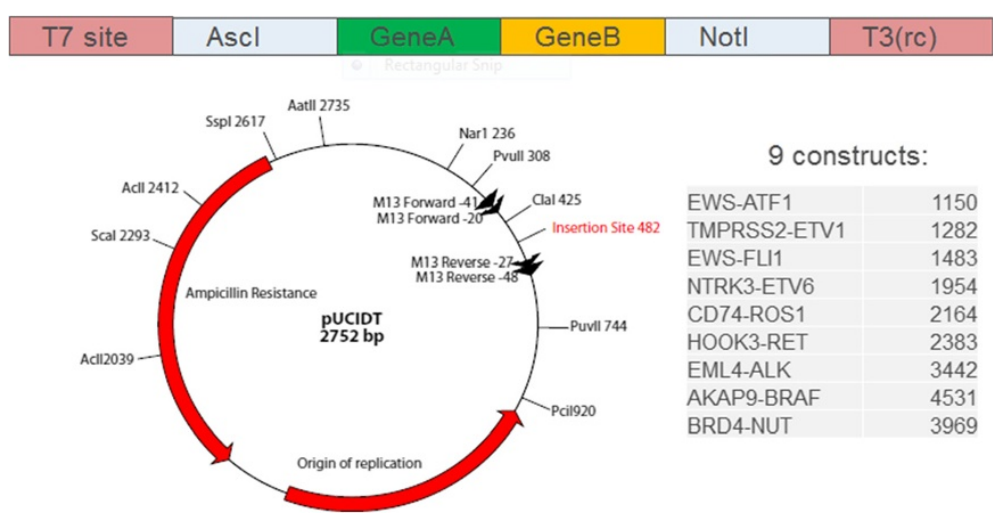

Figure 2 Vector design: the gene sequence was synthesized by IDT and inserted into a pUCIDT vector.

mRNA LT Sample Preparation Kit and sequenced on an Illumina HiSeq $2500(2 \times 101$ cycles $)$.

\section{Bioinformatics}

Illumina sequencing data was converted to FASTQ format using Casava pipeline followed by read quality assessment using FASTQC tool (http://www.bioinformatics.babraham. ac.uk/projects/fastqc/). We analyzed the data using three fusion detection tools: ChimeraScan [11], Tophat-Fusion [12], and Snowshoes-FTD [13] (hereafter referred as CHS, THF, and SSH respectively). The command-line parameters are described in Figure 3. For each analysis tool, we captured the number of sequencing reads supporting each of the nine SGFRs at various abundances (Additional file 1: Table S2), and this table was used for all subsequent analyses. In addition to the nine SGFRs, fusions endogenous to COLO829 were also detected by the analyses. We were able to confirm one endogenous fusion OIP5-NUSAP1 in independent wet-lab validation (Additional file 1: Table S3), although all callers did not identify it. Since endogenous fusions are out of scope of this study, they are not discussed further in this manuscript and we did not attempt to validate in wet-lab every predicted endogenous fusion. However, a parallel sample run with no SGFRs added showed zero reads mapping to the regions of select fused gene junctions, and therefore the COLO829 can be considered to be a high complexity neutral background sample for this study.

\section{Results and discussion}

Analytically, gene fusions are typically detected from RNA-seq data by: 1) Aligning reads to a reference genome or transcriptome assembly; 2) Identifying discordant read pairs, i.e., pairs for which genomic distance between the two ends' alignments is significantly different from the expected genomic distance based on library preparation;

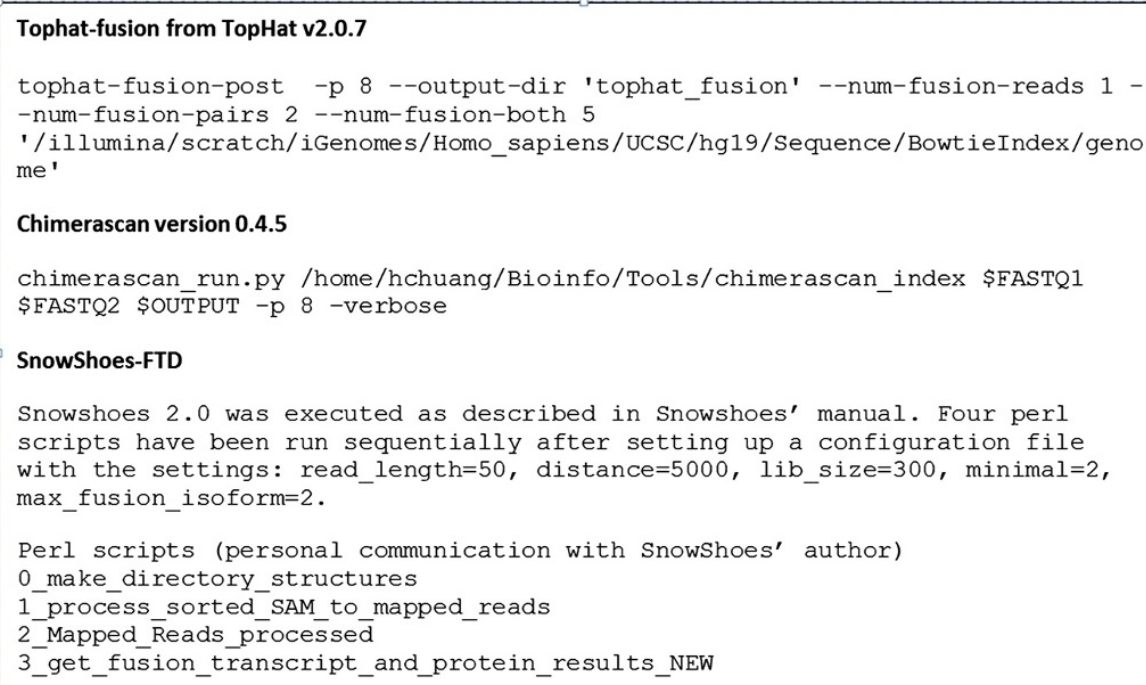

Figure 3 Command-line parameters used for running the three fusion detection tools. Reference genome was GRCh37. In each case, custom scripts were developed internally to extract statistics about fusion-supporting reads. 
3) Extracting split sections of the same read that align to different regions of the genome, thereby, indicating a potential fusion; 4) Algorithm-specific additional steps, such as contig construction, sequence homology search, guided analyses based on exon junction annotation files, etc.

We emphasize here that our focus is to demonstrate utility of the SGFR constructs for evaluating assay performance and to make them available to the clinical and research communities to further active research in gene-fusion detection methods. To that end, the choice of three representative algorithms and the analysis framework is based on our experience in analyzing such data. Since emphasis is on making RNA-seq gene fusion data publically available, we do not attempt to provide a detailed comparative assessment, pros-cons, or performance characterization of the growing number of gene fusion detection tools discussed elsewhere $[14,15]$. However, to highlight the differences in the underlying analytical methods in these three fusion-detection tools, we briefly describe each of the approaches and direct readers to bibliography [11-13] for complete details. THF builds on Tophat to align RNA-seq reads using Bowtie [16] without using any annotation to independently align paired end reads, followed by segment mapping of unaligned reads that are used together for identifying candidate fusion junctions. Next, spliced fusion contig index is created and read segments are remapped using BLAST (in the TophatFusionPost step) followed by stitching all segments together into full read alignments that are further filtered based on criteria, such as number of fusion-supporting reads. SSH uses 50-bp reads that are aligned by BWA [17] guided by customized exon annotation file to identify potential fusions as well as unmapped reads. In our SSH analysis, we retained the first 50-bases from FASTQ files, and SnowShoes-FTD authors provided the annotation file (personal communication). Subsequent steps consists of using Megablast and a junction database to identify overlapping, spanning, and split reads to detect fusions that are further filtered using SnowShoes-FTD author provided false positive list. CHS uses known junctions from an annotation file that guides Bowtie alignment algorithm to find discordant read pairs and unmapped reads. Trimmed unmapped reads are aligned and used in conjunction with previous alignments to identify chimeric events by examining exon junctions from the annotation file. Thus, the three methods share an overall approach of identifying fusions based on aligning paired-end reads and detecting evidence of fusion junction. However, they are different with respect to the specific underlying alignment algorithm, read length, guidance from optionally provided annotation file, postalignment processing to assemble fusion contigs, and parameters used to retain fusions from candidate fusions.

We also verified by running a separate parallel sample that the COLO-829 cell line provided a neutral background, i.e., it did not contain any of the nine SGFRs. Therefore, SGFRs in our experiment were not barcoded prior to spiking into the total RNA. However, barcoded SGFRs should be preferred in other cell lines to avoid mixing of spiked-in fusions and potential endogenous fusions.

Figure 4 demonstrates that at higher abundances, the relationship between number of detected fusions reads and abundance is linear. At lower abundances, the plateaued response might indicate high noise to signal ratio. To verify that fusion reads were present in the original data (true positive signal), we used GSNAP [18] as an independent tool to align entire data against a combined concatenated reference sequence consisting of human genome build GRCh37 and the nine synthetic fusions transcripts. Figure 5 shows the number of fusion-supporting reads identified by GSNAP (blue squares) along with those identified by the three gene fusion detection tools (triangles).

To compare experimental replicates, we calculated the Pearson correlation between number of fusion-supporting reads between replicates (Figure 6) by dividing the data into high read count $(>100)$ and low read count $(<=100)$ groups chosen based on visual inspection of data for illustration purposes. For high read-counts, correlation between replicates' reads for each tool as well as all reads combined together was high (CHS: 0.9613, THF: 0.9990, SSH: 0.9986, All: 0.9955). For low read-counts, corresponding correlation values were lower (CHS: 0.3209, THF: 0.2577, SSH: 0.7292, and All: 0.4025). This indicates higher difference between replicates at lower abundance values that should also translate to more differences in detected fusions at lower abundances. Figure 7 depicts the variability (Y-axis) in number of fusions reads against various abundances (X-axis). For each abundance, variance of the fraction of reads supporting each fusion from the total number of fusion-supporting reads was calculated when at least five out of nine, i.e., more than half, fusions had supporting reads. Clearly, at higher abundances (approximately $6 \mathrm{pMol}$ or higher), variance is consistently low and replicates have almost equal variance indicated by overlapping data points.

To observe the effect of changing minimum number of reads required to call a fusion, Figure 8 depicts the number of fusions detected for each replicate at different minimum reads thresholds. Implicitly, Figure 8 also captures gene-fusion detection sensitivity as the ratio of number of detected fusions to the nine known fusions at various abundances for different minimum number of fusion-supporting reads threshold. For example, at 3.47 pMol, TophatFusion identifies all but the TMPRSS2ETV1 fusion, with a sensitivity value of $8 / 9=88.88 \%$. Sensitivity of replicates is highly similar, except for aberrations in the low abundance zones, and it consistently reaches high values at higher abundance. Since true negatives are unknown, specificity calculation is left as an open question. 

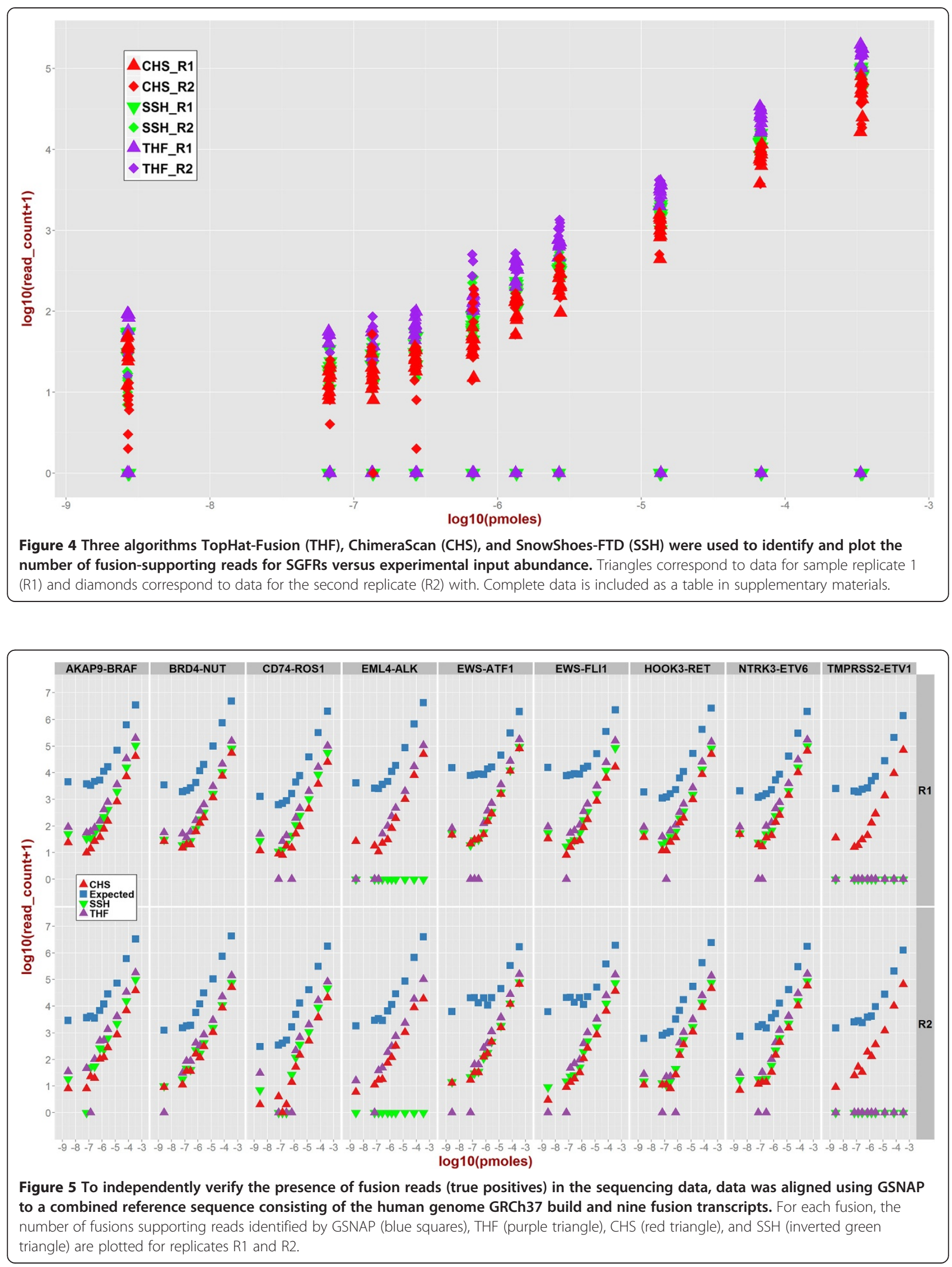

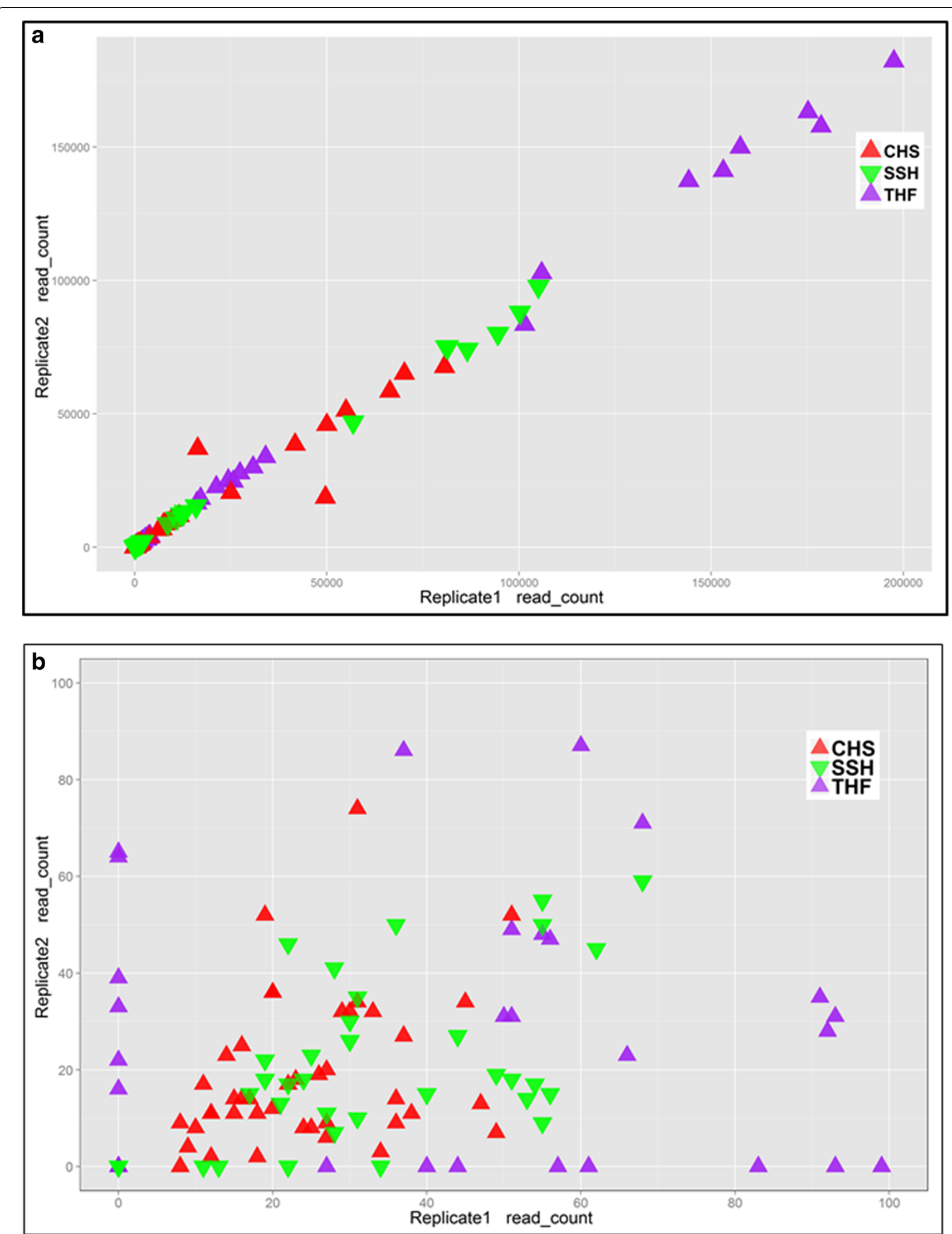

Figure 6 Correlation between replicates based on number of fusion supporting reads. Panel (a) shows fusion-supporting reads (X-axis: Replicate 1, Y-axis: Replicate 2) for high read count (>100). Pearson correlation was CHS: 0.9613, THF: 0.9990, SSH: 0.9986, All: 0.9955. Panel (b) shows data for low read count (<=100) with Pearson correlation values CHS: 0.3209, THF: 0.2577, SSH: 0.7292, All: 0.4025. 

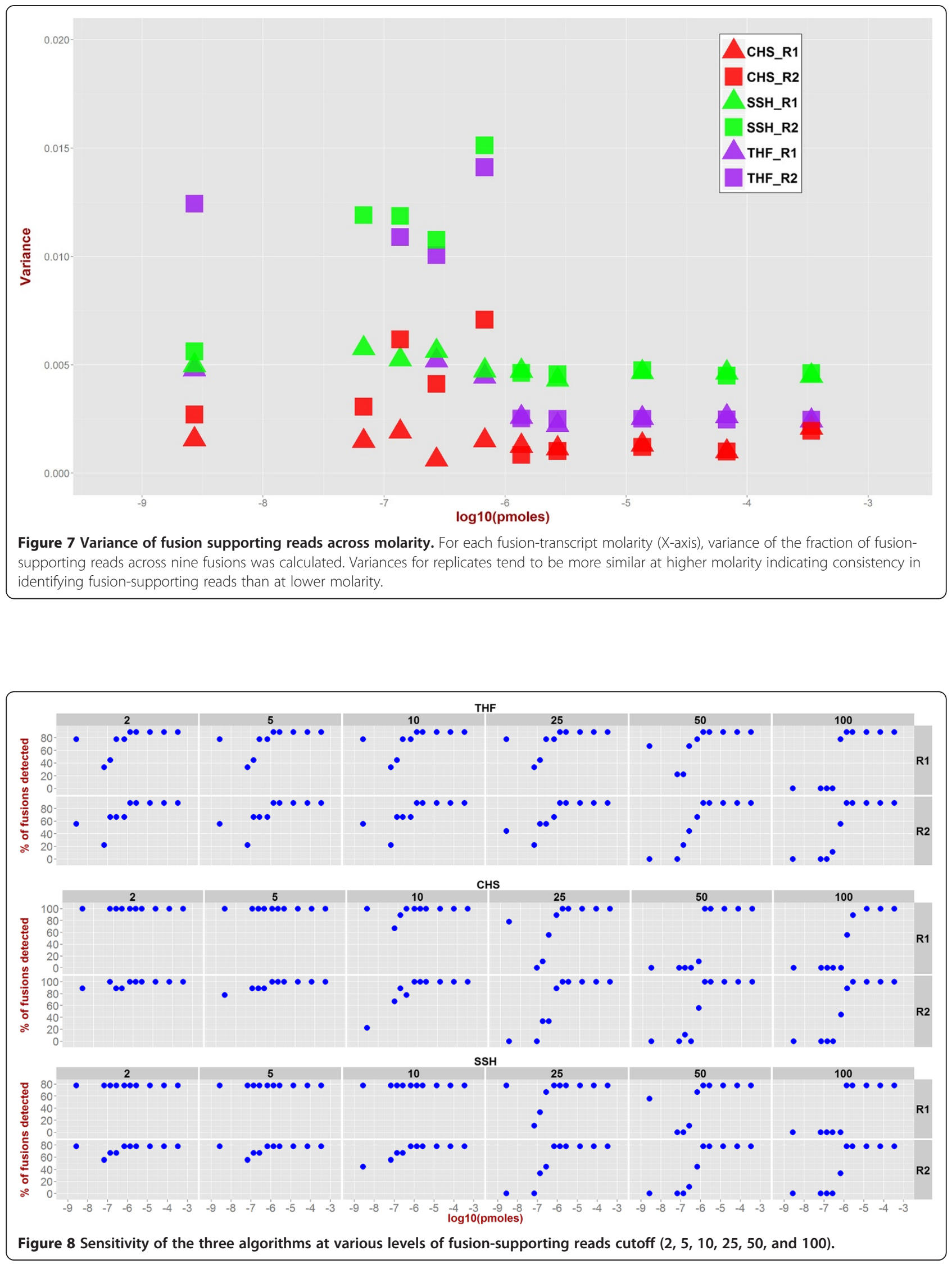
Figure 9 provides in a matrix form a more granular view of detected fusions (brown cells) and undetected fusions (blue cells) at example cut-offs of 2 and 50 fusionsupporting reads. At the minimum read threshold of 2 (Figure 9, left panel), a fusion was either detected or not detected in both replicates in 93\% of the cases. BRD4NUT (undetected in 1.5\% cases) and TMPRSS2-ETV1 (undetected in 66\% cases) marked the two extremes of detectability. None of the SGFRs was unambiguously detected across all molarities by all tools even at an extremely generous cut-off of minimum two fusionsupporting reads. This highlights the challenge in assessing performance metrics with a small set of synthetic constructs-even at the highest abundance in our experiments, $100 \%$ concordant results were not obtained for all of the SFGRs. The data are less reproducible at lower abundances. This indicates that for applications where specific fusion sequences are being investigated, additional constructs may be added to provide quantitative data that is specific for the sequence of interest.

Notably, some fusions were not detected by one or more tool(s) irrespective of molarity as shown by the points on $\mathrm{X}$-axis in Figure 4. As shown in Figure 5, irrespective of the fusion transcript abundance all three tools detected EWS-ATF1, two tools detected EML4-ALK, and only one tool detected TMPRSS2-ETV1. On further investigation of SSH workflow, we discovered that fusion-supporting reads for both EML4-ALK and TMPRSS2-ETV1 were present in the initial candidate fusion list. However, these fusions were subsequently discarded by the SSH workflow when final list of fusions was reported. As end-users of the tool, we could not precisely identify specific reasons for this filtering out and a detailed investigation of $\mathrm{SSH}$ algorithm implementation is out of scope of this study. To explore why THF did not report TMPRSS2-ETV1 fusion, we extracted known fusion-supporting reads from GSNAP alignments and searched for those in the alignment files (generally known as accepted_hits.bam) generated by THF. We discovered that several fusionsupporting reads were aligned against TMPRSS2 (chr21:42.84-42.9 mb) and ETV1 (chr7:13.93-14.03 mb) loci across various molarities as shown in Additional file 1: Table S4. However, TMPRSS2-ETV1 fusion was not reported in the final list of fusions after the TophatFusionPost step was executed. A detailed investigation of actual THF algorithm implementation and specific reasons behind filtering out the fusion is out of scope of this study. However, observations based on additional investigation of unreported fusions highlight the critical importance of tool-specific criteria and parameters that might lead to false negatives or false positives-evidence for fusions from alignment data was processed differently by different tools yielding different results.

For the sake of completeness, we also note that each detection tool has a large number of input parameters that significantly affect its detection ability. Figure 4 depicts overall trend in capturing fusion-supporting reads based on our experimental design and chosen parameters. However, assessing the dynamic range and limits of detection for analytical tools will require extensive combinatorial selection of parameters, an in-depth analysis of algorithm implementation, and a much larger number of SGFRs
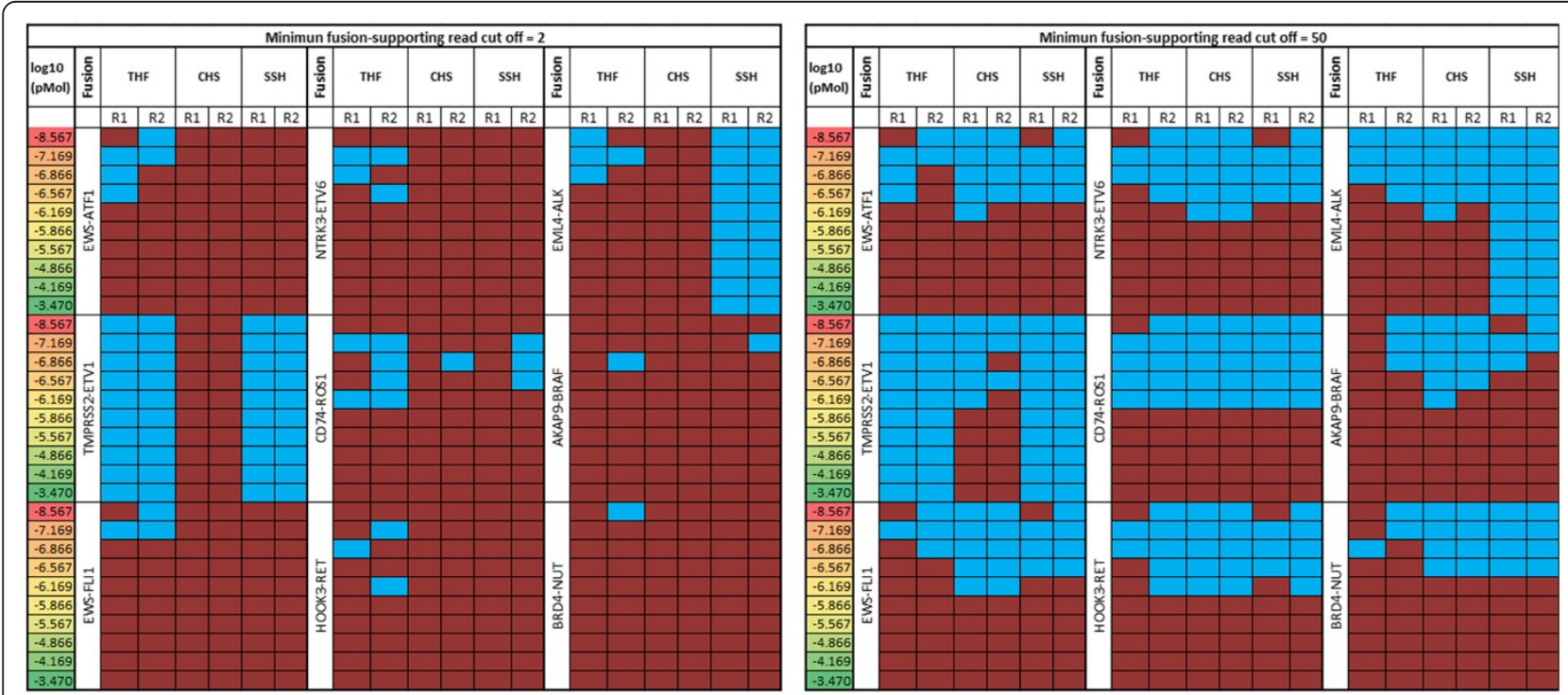

Figure 9 Fusions detected by each algorithm. For two example thresholds of 2 (left matrix) and 50 (right matrix) on minimum number of fusion-supporting reads, number of fusions detected at different concentrations for two replicates R1 and R2 are shown. Brown cell: fusion detected. Blue cell: fusion missed. For example, at minimum threshold of 2, BRD4-NUT was positively identified most frequently (59/60 times) and TMPRSS2-ETV1 was detected least frequently (20/60 times). 
across wide range of transcript abundance as part of testing and validation. These are out of scope of this study that is primarily focused on making available a publically available data for collaborative research and highlighting some of the issues in RNA-seq based gene fusion detection based on our analysis framework.

\section{Conclusion}

The key contribution of this work is the first publicly available gene fusion RNA-seq data that specifically targets known oncogenic gene fusions that are gaining increasing importance in clinical genomics based on next-generation sequencing. The community can augment this dataset and the proposed analytical framework to further collaborative development of advanced analytical tools for gene fusion detection from RNA-seq data.

\section{Data availability}

All sequencing data is available in FASTQ format from the Short Read Archive under accession number SRP043081.

\section{Additional file}

Additional file 1: Table S1. Fusion sequences. Table S2. Fusion read counts across all samples. Table S3. Endogenous fusions. Table S4. Fusion-supporting reads from TMPRSS2-ETV1 Tophat Fusion analysis.

\section{Competing interests}

WDT, WSL, CL, SW, DWC, and JDC declare that they have no competing interests. At the time this work was conducted, SJKP, H-YC, NK, VM, and TKM were salaried employees and shareholders of Illumina, Inc.

\section{Authors' contributions}

SJKP, TKM, and JDC led gene-fusion sequence selection, vector design, and library development. SJKP, WSL, SW, NK, VM, JDC led wet lab methods and sequencing. WDT, H-YC, DWC conceptualized bioinformatics and analytical framework. WDT, $\mathrm{CL}$, and $\mathrm{H}-\mathrm{YC}$ carried out analyses, comparisons, customized script development, data tabulation-compilation, and figure generation. WDT and $C L$ uploaded data to SRA. WDT and SJKP led manuscript development. TKM, DWC, and JDC guided this TGen-Illumina collaborative study. All authors participated in manuscript revisions. All authors read and approved the final manuscript.

\section{Acknowledgements}

Research partially supported by a Stand Up To Cancer - Melanoma Research Alliance Melanoma Dream Team Translational Cancer Research Grant (\#SU2C-AACR-DT0612). Stand Up To Cancer is a program of the Entertainment Industry Foundation administered by the American Association for Cancer Research. The authors thank TGen's IT division for computational resources.

\section{Author details}

${ }^{1}$ Translational Genomics Research Institute (TGen), 445 N 5th Street, SUITE 600, Phoenix, AZ 85004, USA. ${ }^{2}$ Illumina, Inc, San Diego, CA, USA.

\section{Received: 21 April 2014 Accepted: 24 September 2014}

Published: 30 September 2014

\section{References}

1. Villanueva MT: Genetics: gene fusion power. Nat Rev Clin Oncol 2012, 9:188.

2. Goldman JM, Melo JV: Chronic myeloid leukemia-advances in biology and new approaches to treatment. N Engl J Med 2003, 349:1451-1464.
3. Saglio G, Morotti A, Mattioli G, Messa E, Giugliano E, Volpe G, Rege-Cambrin G, Cilloni D: Rational approaches to the design of therapeutics targeting molecular markers: the case of chronic myelogenous leukemia. Ann N Y Acad Sci 2004, 1028:423-431.

4. Kakizuka A, Miller W, Umesono K, Warrel R, Franekl S, Murty V, Dmitrovsky E, Evans R: Chromosomal translocation $\mathrm{t}(15 ; 17)$ in human acute promyelocytic leukemia fuses RAR alpha with a novel putative transcription factor, PML. Cell 1991, 66:663-674.

5. Huang ME, Ye YC, Chen SR, Cai JR, Lu JX, Zhoa L, Gu LJ, Wang ZY: Use of all-trans retinoic acid in the treatment of acute promyelocytic leukemia. Blood 1988, 72:567-572.

6. Castaigne $S$, Chomienne C, Daniel M, Ballerini P, Berger R, Fenaux P, Degos L: All-trans retinoic acid as a differentiation therapy for acute promyelocytic leukemia: I. Clinical results. Blood 1990, 76:1704-1709.

7. Gerber DE, Minna JD: ALK inhibition for non-small cell lung cancer: from discovery to therapy in record time. Cancer Cell 2010, 18:548-551.

8. Ou SH, Bazhenova L, Camidge DR, Solomon BJ, Herman J, Kain T, Bang YJ, Kwak EL, Shaw AT, Salgia R, Maki RG, Clark JW, Wilner KD, lafrate AJ: Rapid and dramatic radiographic and clinical response to an ALK inhibitor (crizotinib, PF02341066) in an ALK translocation-positive patient with non-small cell lung cancer. J Thorac Oncol 2010, 5:2044-2046.

9. Kwak EL, Bang YJ, Camidge DR, Shaw AT, Solomon B, Maki RG, Ou SH, Dezube BJ, Jänne PA, Costa DB, Varella-Garcia M, Kim WH, Lynch TJ, Fidias P, Stubbs H, Engelman JA, Sequist LV, Tan W, Gandhi L, Mino-Kenudson M, Wei GC, Shreeve SM, Ratain MJ, Settleman J, Christensen JG, Haber DA, Wilner K, Salgia R, Shapiro Gl, Clark JW, et al: Anaplastic lymphoma kinase inhibition in non-small-cell lung cancer. N Engl J Med 2010, 363:1693-1703.

10. Pleasance ED, Cheetham RK, Stephens PJ, McBride DJ, Humphray SJ, Greenman CD, Varela I, Lin ML, Ordóñez GR, Bignell GR, Ye K, Alipaz J, Bauer MJ, Beare D, Butler A, Carter RJ, Chen L, Cox AJ, Edkins S, KokkoGonzales PI, Gormley NA, Grocock RJ, Haudenschild CD, Hims MM, James T, Jia M, Kingsbury Z, Leroy C, Marshall J, Menzies A, et al: A comprehensive catalogue of somatic mutations from a human cancer genome. Nature 2010, 463:191-196.

11. Iyer MK, Chinnaiyan AM, Maher CA: ChimeraScan: a tool for identifying chimeric transcription in sequencing data. Bioinformatics 2011, 27:2903-2904.

12. Kim D, Salzberg SL: TopHat-fusion: an algorithm for discovery of novel fusion transcripts. Genome Biol 2011, 12:R72.

13. Asmann YW, Hossain A, Necela BM, Middha S, Kalari KR, Sun Z, Chai HS, Williamson DW, Radisky D, Schroth GP, Kocher JP, Perez EA, Thompson EA: A novel bioinformatics pipeline for identification and characterization of fusion transcripts in breast cancer and normal cell lines. Nucleic Acids Res 2011, 39:e100.

14. Carrara M, Beccuti M, Lazzarato F, Cavallo F, Cordero F, Donatelli S, Calogero RA: State-of-the-art fusion-finder algorithms sensitivity and specificity. Biomed Res Int 2013, 2013:340620

15. Wang Q, Xia J, Jia P, Pao W, Zhao Z: Application of next generation sequencing to human gene fusion detection: computational tools, features and perspectives. Brief Bioinform 2013, 14:506-519.

16. Langmead B, Salzberg SL: Fast gapped-read alignment with Bowtie 2 . Nat Methods 2012, 9:357-359.

17. Li H, Durbin R: Fast and accurate long-read alignment with BurrowsWheeler transform. Bioinformatics 2010, 26:589-595.

18. Wu TD, Nacu S: Fast and SNP-tolerant detection of complex variants and splicing in short reads. Bioinformatics 2010, 26:873-881.

doi:10.1186/1471-2164-15-824

Cite this article as: Tembe et al:: Open-access synthetic spike-in mRNAseq data for cancer gene fusions. BMC Genomics 2014 15:824. 